\title{
COCHRANE CORNER \\ Valproic acid, valproate and divalproex in the maintenance treatment of bipolar disorder ${ }^{\dagger}$
}

\author{
Andrea Cipriani, Keith Reid, Allan H. Young, Karine Macritchie, John Geddes
}

${ }^{\dagger}$ This review is an abridged version of a Cochrane Review previously published in the Cochrane Database of Systematic Reviews, 2013, Issue 10, doi: 10.1002/14651858. CD003196.pub2 (see www. thecochranelibrary.com for information). Cochrane Reviews are regularly updated as new evidence emerges and in response to feedback, and the Cochrane Database of Systematic Reviews should be consulted for the most recent version of the review.

We thank the Cochrane Review Group for their support in publishing these reviews.

See commentary on pp. $3-4$, this issue.

\section{Background}

Bipolar disorder is a recurrent illness that is amongst the top 30 causes of disability worldwide and is associated with significant healthcare costs. In the past, emphasis was placed solely on the treatment of acute episodes of bipolar disorder; recently, the importance of episode prevention and of minimisation of iatrogenicity has been recognised. For many years, lithium was the only mood stabiliser in common use, and it remains an agent of first choice in the preventative treatment of bipolar disorder. However, an estimated $20 \%$ to $40 \%$ of patients may not respond adequately to lithium. Valproate is an anticonvulsant drug that has been shown to be effective in acute mania and is frequently used in maintenance treatment of bipolar disorder. When the acceptability of long-term treatment is considered, together with efficacy, the adverse event profile of a medication is also important. This is an update of a Cochrane review first published in 2001 and last updated in 2009

\section{Objectives}

1. To determine the efficacy of valproate continuation and maintenance treatment: (a) in preventing or attenuating manic, depressive and mixed episodes of bipolar disorder; (b) in preventing or attenuating episodes of bipolar disorder in patients with rapid cycling disorder; and (c) in improving patients' general health and social functioning, as measured by global clinical impression, employment and marital stability. 2. To review the acceptability to patients of long-term valproate treatment, as measured by numbers of dropouts and reasons for dropping out, by compliance and by reference to patients' expressed views regarding treatment. 3 . To investigate the adverse effects of valproate treatment (including general prevalence of sideeffects) and overall mortality rates.

\section{Search methods}

Search of the Cochrane Register of Controlled Trials and the Cochrane Depression, Anxiety and Neurosis Group Register (CCDANCTR) (to January 2013), which includes relevant randomised controlled trials from the following bibliographic databases: The Cochrane Library (all years), EMBASE (1974 to date), MEDLINE (1950 to date) and PsycINFO (1967 to date). No language restrictions were applied. Reference lists of relevant papers and previous systematic reviews were handsearched. Pharmaceutical companies marketing valproate and experts in this field were contacted for supplemental data.

Selection criteria

Randomised controlled trials allocating participants with bipolar disorder to long-term treatment with valproate or any other mood stabiliser, or antipsychotic drugs, or placebo. Maintenance treatment was defined as treatment instituted specifically or mainly to prevent further episodes of illness.

Data collection and analysis

Three review authors independently extracted data. A doubleentry procedure was employed by two review authors.
Information extracted included study characteristics, participant characteristics, intervention details and outcome measures in terms of efficacy, acceptability and tolerability. For dichotomous data, risk ratios were calculated with $95 \%$ confidence intervals (Cls). For statistically significant results, we calculated the number needed to treat for an additional beneficial outcome (NNTB) and the number needed to treat for an additional harmful outcome (NNTH). For continuous data, mean differences (MDs) or standardised mean differences (SMDs) were calculated along with 95\% Cls. MDs were used when the same scale was used to measure an outcome; SMDs were employed when different scales were used to measure the same outcome. The primary analysis used a fixed-effect model. Binary outcomes were calculated on a strict intention-to-treat (ITT) basis; dropouts were included in this analysis. When data were missing and the method of 'last observation carried forward' (LOCF) had been used to do an ITT analysis, then the LOCF data were used.

\section{Main results}

Six randomised controlled trials (overall 876 participants) lasting 6 to 24 months were included. Two studies (overall 312 participants) compared valproate with placebo, four studies (overall 618 participants) valproate with lithium, one study (overall 23 participants) valproate with olanzapine and one study (overall 220 participants) valproate with the combination of valproate plus lithium. In terms of study quality, most studies reported the methods used to generate random sequences; however, only one study reported enough details on allocation concealment. Four of six included studies described their design as 'double blind', but only two trials reported full details about blinding. Valproate was more effective than placebo in preventing study withdrawal due to any mood episode (RR 0.68, 95\% Cl 0.49 to 0.93; NNTB 8), but no difference in efficacy was found between valproate and lithium (RR 1.02, $95 \% \mathrm{Cl} 0.87$ to 1.20$)$. Valproate was associated with fewer participants dropping out of treatment for any cause when compared with placebo or lithium (RR $0.82,95 \% \mathrm{Cl} 0.71$ to 0.95 and $\mathrm{RR} 0.87,95 \% \mathrm{Cl} 0.77$ to 0.98 respectively). However, combination therapy with lithium plus valproate was more likely to prevent relapse than was monotherapy with valproate (RR 0.78, 95\% Cl 0.63 to 0.96). Significant differences in adverse event frequencies were found: lithium was associated with more frequent diarrhoea, polyuria, increased thirst and enuresis, whereas valproate was associated with increased sedation and infection.

\section{Authors' conclusions}

Limited evidence supports the efficacy of valproate in the long-term treatment of bipolar disorder. Clinicians and patients should consider acceptability and tolerability profile when choosing between lithium and valproate-their combination or other agents-as long-term treatment for bipolar disorder.

Assessed as up to date: 11 Jan 2013 